\title{
Gemcitabine delivered by fucoidan/chitosan nanoparticles presents increased toxicity over human breast cancer cells
}

\author{
Catarina Oliveira1,2, Nuno M Neves,2,3, Rui L Reis ${ }^{1,2,3}$, Albino Martins ${ }^{1,2}$ \& Tiago H Silva*,1,2 \\ 13B's Research Group - Biomaterials, Biodegradables \& Biomimetics, University of Minho, Headquarters of the European Institute \\ of Excellence on Tissue Engineering \& Regenerative Medicine, AvePark, Parque de Ciência e Tecnologia, 4805-017 Barco, \\ Guimarães, Portugal \\ 2ICVS/3B's, PT Government Associate Laboratory, Braga/Guimarães, Portugal \\ ${ }^{3}$ The Discoveries Centre for Regenerative \& Precision Medicine, Headquarters at University of Minho, Avepark, $4805-017$ Barco, \\ Guimarães, Portugal \\ *Author for correspondence: tiago.silva@dep.uminho.pt
}

\begin{abstract}
Aim: To produce marine-origin nanoparticles (NPs) aiming to develop more effective and tolerated therapies for breast cancer. Materials \& methods: NPs based in two marine-origin polymers (fucoidan and chitosan) were prepared by polyelectrolyte complexation, for the delivery of an antitumor drug model (gemcitabine [Gem]). Results: Final formulation resulted in stable NPs around 115-140 nm in size and with a polydispersity index less than 0.2 . Gem was encapsulated at a maximum entrapment efficiency of $35-42 \%$. Drug-release studies demonstrated that around $84 \%$ of Gem is released within $4 \mathrm{~h}$. Cytotoxicity results of Gem-loaded NPs showed increased toxicity (around 25\%) when compared with free Gem. Conclusion: The drug-loaded NPs present increased toxicity over human breast cancer cells without increasing toxic effects over endothelial cells.
\end{abstract}

First draft submitted: 4 January 2018; Accepted for publication: 11 May 2018; Published online: 7 September 2018

Keywords: breast cancer cells • chitosan • drug delivery system • endothelial cells • fucoidan • gemcitabine • nanoparticles $\bullet$ polyelectrolyte complexation

Cancer is one of the leading causes of death worldwide and is characterized by the uncontrolled and fast growth of cells. In 2012, there were around 14 million new cases of cancer and around 8 million resulted in death. By 2035, it is expected to be around 24 million new cases per year [1]. Breast cancer is the most common cancer in females and the second deadliest [2]. Surgery, radiation and systemic therapies (chemotherapy, hormonal and target) are some of the current breast cancer treatment modalities available, depending on the extent and type of cancer [3]. Some of these treatments may present some limitations since, besides affecting the tumor, they also affect the surrounding healthy environment, and may present some side effects [3,4]. For these reasons, the use of natural substances is an interesting alternative due to their low toxicity and biological and chemical similarities to native tissue compositions.

Among natural substances, marine organisms are valuable sources of materials with intriguing properties and characteristics [5]. Fucoidan $(\mathrm{Fu})$ is a naturally derived sulfated polysaccharide extracted from brown seaweed and has been extensively investigated because of its various biological properties such as anticoagulant, antiviral, antiangiogenic, anti-inflammatory, immunomodulating and, most recently, antitumor behavior [6,7]. Looking specifically at its antitumoral behavior, we recently reported that different Fu extracts present different biological responses over human breast cancer and noncancer cell lines with chemical structure apparently playing an important role [8]. Some Fu extracts presented the desired antitumoral behavior, whereas other presented toxicity for both cancer and noncancer cells or where nontoxic for both types of cells, depending on their chemical features.

In this work, we propose the use of nontoxic Fu to develop a natural marine-origin drug delivery system (DDS) for the release of anticancer drugs. Indeed, Fu have been included in nanosystems for diagnostic, drug delivery and regenerative medicine $[9,10]$ and have been combined with chitosan $(\mathrm{CH})$, a marine-derived polycation, to prepare 
nanoparticles (NPs) by polyelectrolyte complexation for the delivery of growth factor drugs and other biological compounds [10-13].

NPs have been developed as DDS for the delivery of drugs or biomolecules. NPs-based DDS presents attractive properties since they can pass through the smallest capillary vessels and penetrate cells/tissue gaps to arrive at the target site $[14,15]$. They may also improve macromolecule stability, protecting them from enzymatic degradation and controling their release profile. However, they may present some limitations like drug loss during circulation and limited drug-loading capacity.

Gemcitabine (Gem), an antitumor drug, has been applied in the treatment of different cancers such as lung, ovary and pancreatic [16-18]. It has been also used in the treatment of breast cancer, as a single therapy or in combination with other chemotherapeutic agents $[19,20]$. Gem is a nucleoside analog that inhibits DNA synthesis, by its incorporation into DNA, followed by inhibition of cell proliferation. Gem presents a short biological halflife, being rapidly metabolized. This limits its biological effectiveness, since higher doses are required to reach a therapeutic concentration, increasing the risk of side effects [21,22]. In this sense, there is the unmet therapeutic need to try to enhance Gem biological response by incorporating it into a DDS, which has been previously reported [23,24].

To the best of our knowledge, there are no reports in the literature regarding the development of $\mathrm{Fu} / \mathrm{CH}$ NPs for the delivery of Gem. In this sense, we propose the combination of Fu (negatively charged) with $\mathrm{CH}$ by polyelectrolyte complexation to produce NPs for controlled release of Gem as potential breast cancer therapy. Regarding NP production, there are different parameters that need to be optimized such as polymers concentration and ratio, polymeric solution $\mathrm{pH}$ and ionic strength. Therefore, these different parameters were tested and combined to obtain the NPs with smaller size and polydispersity index (PDI). NPs stability is another important aspect that needed to be evaluated. Keeping in mind the tumor microenvironment, NPs toxicity was evaluated over cancer and noncancer cells. The release profile of Gem was analyzed and loaded-NPs tested over endothelial and breast cancer cells as an in vitro assessment of antitumor efficiency through cytotoxicity.

\section{Experimental section}

Materials

Fu from Fucus vesiculosus (molecular weight [MW] by gel permeation chromatography [GPC] 45-75 kDa) was purchased from Marinova (batch number: DPFVF2015505), whereas CH 95/20 (MW by GPC 40-150 kDa) was purchased from Heppe Medical Chitosan GmbH (batch number: 212-261115-04, Halle, Germany). 1[3-(Dimethylamino)propyl]-3-ethylcarbodiimide hydrochloride (EDC) and $N$-hydroxysuccinimide (NHS) were both purchased from Sigma-Aldrich (MO, USA). Centrifugal concentrators Vivaspin $20300 \mathrm{KD}$ (Sartorius; Stonehouse, UK) were purchased from Fisher Scientific. 2'-Deoxy-2',2'-difluorocytidine (Gem - $\left.\mathrm{C}_{9} \mathrm{H}_{11} \mathrm{~F}_{2} \mathrm{~N}_{3} \mathrm{O}_{4}\right)$ was purchased from Carbosynth (Berkshire, UK). Human umbilical vein endothelial cells (EA.hy926) and breast cancer cells (MDA-MB-231) were both purchased from ATCC (VA, USA).

\section{Methods}

Preparation of NPS

Marine-origin polymeric NPs were prepared by polyelectrolyte complexation. Optimization was performed to study the effect of different parameters over size and PDI. From the results obtained, for each parameter, we chose the production condition that combined the smaller size and PDI values. Different parameters were evaluated, such as concentration of both polymers (ranging from 0.25 to $1 \mathrm{mg} / \mathrm{ml}$ ); the ratio of polymers solution $(\mathrm{CH}: \mathrm{Fu}-1: 2$, 1:1, 2:1); $\mathrm{CH}$ and $\mathrm{Fu}$ solutions $\mathrm{pH}$; ionic strength (ultrapure water [UPW], osmotized water and $0.9 \% \mathrm{NaCl}$ ) and cross-linking agent concentrations: 600/240, 300/120 and 150/60 mM (EDC/NHS). Each parameter was evaluated separately while all the others were kept constant.

After all the optimization steps, NP parameters were maintained for all further assays. Briefly, $\mathrm{CH}$ was dissolved in $1 \% \mathrm{v} / \mathrm{v}$ acetic acid solution at $0.5 \mathrm{mg} / \mathrm{ml}$ concentration, whereas the polyanion $(\mathrm{Fu}$ ) was prepared in UPW to reach a final concentration of $0.5 \mathrm{mg} / \mathrm{ml}$. CH and Fu solutions were adjusted with $1 \mathrm{M} \mathrm{HCl}$ and $1 \mathrm{M} \mathrm{NaOH}$, depending on the desired $\mathrm{pH}$. $\mathrm{CH}$ solution was adjusted to $\mathrm{pH}$, whereas Fu solution was measured and the $\mathrm{pH}$ adjusted if its values were not as expected $(\mathrm{pH} 9) .3 \mathrm{nl}$ of Fu solution $(0.5 \mathrm{mg} / \mathrm{ml})$ was added, using a precision syringe pump, to $3 \mathrm{ml}$ of $\mathrm{CH}$ solution $(0.5 \mathrm{mg} / \mathrm{ml})$ leading to a final theoretical ratio $(\mathrm{w} / \mathrm{w})$ of $1: 1$ and a spontaneous formation of NPs occured. Right after NP production, $300 \mathrm{mM} \mathrm{EDC} \mathrm{+} 120 \mathrm{mM}$ NHS were added to the NP solution. NPs were stirred for $2 \mathrm{~h}$ at $600 \mathrm{rpm}$. After that, NPs were collected using $300 \mathrm{kDa}$ vivaspin 
filters for $15 \mathrm{~min}$ at $4000 \mathrm{rpm}$. NPs were washed twice with UPW. Glucose was added in all centrifugation steps to avoid NP aggregation. Finally, NPs were filtered with $0.22 \mu \mathrm{m}$ filters.

\section{Characterization of NPS}

Dynamic light scattering

NP size and PDI were determined by dynamic light scattering (DLS) using a Zetasizer Nano ZS (Malvern Instruments, Malvern, UK). Samples were diluted in UPW and the size analysis lasted $180 \mathrm{~s}$ and performed at $25^{\circ} \mathrm{C}$, in triplicate. The zeta potential measurements were performed by a dip cell in automatic mode.

NP tracking analysis

NP tracking analysis (NTA) experiments were performed using a NanoSight NS500 instrument (Malvern Panalytical, Salisbury, UK). This system includes a charge-coupled device camera that allows visualization and tracking of Brownian motion of laser-illuminated particles in suspension. Video images were analyzed using NTA analytical software version 2.3. The measurements were made at room temperature and each video sequence was captured over $60 \mathrm{~s}$.

Scanning electron microscopy \& scanning transmission electron microscopy

Measurement of particles' size and morphology observation were obtained using SEM (Scanning electron microscopy; Auriga, Carl Zeiss). A drop of NPs solution was placed in the stub for SEM analysis and left to dry overnight. They were then vacuum-coated with a gold-platinum mixture. Scanning transmission electron microscopy (STEM) analysis was also performed with the same equipment.

NPs physiological \& storage stability

To mimic and assess the stability of NPs at physiological conditions, the NPs were diluted in $0.9 \% \mathrm{NaCl}$ and kept at $37^{\circ} \mathrm{C}$. The size and PDI of NPs were measured by DLS every day, until day 7.

To evaluate NPs storage stability after production, NPs were diluted in UPW and kept at $4^{\circ} \mathrm{C}$. NPs size and PDI were measured by DLS every week for up to 2 months.

NPs cytotoxicity

Cell culture \& cell viability

The cytotoxicity of the optimized NPs was evaluated over human umbilical vein endothelial (EA.hy926, cell line) and breast cancer (MDA-MB-231, cell line) cells. Increasing concentration of NPs were used until toxic effects were observed. The CellTiter 96 AQueous One Solution cell proliferation assay (Promega; WI, USA) was performed at 24, 48 and $72 \mathrm{~h}$. At these time points, the culture medium was removed and the testing samples were rinsed with sterile phosphate-buffered saline (PBS). A mixture of culture medium (without Fetal Bovine Serum and phenol red) and MTS reagent (5:1 ratio) was added to each well and left to incubate for $3 \mathrm{~h}$, at $37^{\circ} \mathrm{C}$, in a humidified $5 \% \mathrm{CO}_{2}$ atmosphere. Thereafter, the absorbance of the MTS reaction medium from each sample was read in triplicate at $490 \mathrm{~nm}$ in a microplate reader (Synergy HT, Bio-TEK). All experiments were performed in triplicate. This experiment was repeated with Gem-loaded NPs (NPs_Gem), the amount of free Gem being the same as the amount loaded into the developed NPs.

Drug encapsulation \& release studies

Drug entrapment efficiency

Gem concentrations, ranging from 0.2 to $3.2 \mathrm{mg} / \mathrm{ml}$, were encapsulated into the NPs' optimized formulation. Gem was added to $\mathrm{CH}$ solution and NPs were produced as described in the section titled Preparation of NPs, maintaining the same production conditions ( $\mathrm{CH}: \mathrm{Fu}: \mathrm{Gem}$ ratio 1:1:0.3).

Entrapment efficiency (EE) was calculated by measuring the initial concentration and nonencapsulated Gem, according to the following formula:

$$
\% E E=\frac{(\text { Initial concentration }- \text { non }- \text { entrapped drug })}{\text { initial concentration }} \times 100
$$

The samples were analyzed by UV spectroscopy (Shimadzu) at the maximum absorbance of $268 \mathrm{~nm}$. All the experiments were performed in triplicate. 
Gem-release studies

The release kinetics of Gem was measured by placing $1.5 \mathrm{ml}$ of NPs_Gem into dialysis membranes, and this system into $50 \mathrm{ml}$ of $0.9 \% \mathrm{NaCl}$ at $37^{\circ} \mathrm{C}$ under $60 \mathrm{rpm}$ stirring. At predetermined time periods $(0,5,15,30,45 \mathrm{~min}$; $1,1.5,2,3,4,5,6,8,12,24,48$ and $72 \mathrm{~h}$ ), an aliquot of $2 \mathrm{ml}$ of the release solution was taken and immediately measured at the previously defined absorbance. The experiments were performed in triplicate.

Statistical analysis

Statistical analysis was performed using Graph Pad Prism Software. Differences between the different conditions of the cellular assays were analyzed using nonparametric test (Kruskal-Wallis test), and $\mathrm{p}<0.05$ was considered significant. Data are presented as mean \pm standard deviations.

\section{Results}

\section{Optimization of NPs production parameters}

To produce marine-origin polymeric NPs with constant sizes, several processing parameters were tested, namely polymers concentration and ratios, $\mathrm{pH}$ of the solution, ionic strength and influence of the cross-linker. Experimental results point out that a decrease in polymers concentration leads to the formation of smaller NPs (Figure 1A). With the same trend, the PDI tends to decrease for concentrations down to $0.5 \mathrm{mg} / \mathrm{ml}$. However, for the lowest concentration, the PDI slightly increased indicating that at this polymers concentration the NPs are less homogenous. In this sense, and by the combination of these two important properties, the working concentration was set as $0.5 \mathrm{mg} / \mathrm{ml}$ for both $\mathrm{CH}$ and $\mathrm{Fu}$. When $\mathrm{Fu}$ is in excess ( $\mathrm{CH}: \mathrm{Fu}$ ratio 1:2) in the final formulation, the NPs present a size around $500 \mathrm{~nm}$ and are highly polydispersive (PDI $>0.3$; Figure $1 \mathrm{~B}$ ). For the 2:1 ratio, the NPs present a higher PDI than 1:1. Therefore, this last ratio was used for all further experiments. The influence of $\mathrm{pH}$ solution was evaluated for each polymer separately, maintaining the $\mathrm{pH}$ of the other polymer solution (Figure 1C $\& \mathrm{D})$. For the $\mathrm{CH}$ solution, $\mathrm{pH} 5$ was chosen, whereas for $\mathrm{Fu}$ the testing condition that presented smaller size and PDI values was the one with $\mathrm{pH} 9$ ( $\mathrm{pH}$ of Fu solution). Ionic strength was also evaluated by dissolving the polymers in different solvents with increasing ionic strength: UPW, osmotized water and $0.9 \% \mathrm{NaCl}$. UPW was the condition where the NPs presented the lower PDI and size values (Figure 1E). Taking together, the optimal production parameters were set as: $0.5 \mathrm{mg} / \mathrm{ml}$ of both $\mathrm{CH}$ and $\mathrm{Fu}, 1: 1$ ratio, $\mathrm{pH} 5(\mathrm{CH})$ and $\mathrm{pH} 9(\mathrm{Fu})$ and UPW as the polymers solvent. After setting these parameters, the concentration of a cross-linking agent was evaluated. Different concentrations of EDC/NHS were tested, being the $300 \mathrm{mM}$ of EDC and $120 \mathrm{mM}$ of NHS, the condition that presented the best outcomes in terms of size and PDI (Figure 1F).

\section{NPs characterization \\ NPs stability}

After optimizing all the production parameters, two different conditions were chosen to continue the characterization of the NPs: one without cross-linking (no EDC/NHS) and the other cross-linked with EDC/NHS (300 mM EDC + $120 \mathrm{mM} \mathrm{NHS).} \mathrm{These} \mathrm{two} \mathrm{conditions} \mathrm{were} \mathrm{used} \mathrm{to} \mathrm{perform} \mathrm{stability} \mathrm{assays,} \mathrm{both} \mathrm{at} \mathrm{physiologi-}$ cal conditions $\left(37^{\circ} \mathrm{C}, 0.9 \% \mathrm{NaCl}\right.$; Figure $\left.2 \mathrm{~A}\right)$ and for storage conditions ( ${ }^{\circ} \mathrm{C}$, UPW; Figure $2 \mathrm{~B}$ ). At physiological conditions, both size and PDI increase along the 7 days of the study, presenting values for PDI above 0.3 from day 4 on, concerning noncross-linked NPs. The size of cross-linked NPs was stable along the 7 days, whereas the PDI presents some slightly variations in some time points, these values being below 0.2 . For the storage condition, both types of NPs present no significant variations in terms of size and PDI for up to 2 months.

\section{NP properties}

Based on stability results, cross-linked NPs were further characterized. NPs present a size of $140 \mathrm{~nm}$, a PDI = 0.172 and a zeta potential of $29 \mathrm{mV}$ by DLS (Table 1). NPs were also evaluated by NTA, presenting a size around $116 \mathrm{~nm}$ and a final concentration of $7.81 \times 10^{10} / \mathrm{ml}$.

Nanoparticle morphology and size in dry state were also observed by SEM and STEM (Figure 3A \& B). NPs presented a round shape and a size smaller than $100 \mathrm{~nm}$ in both microscopic analyses.

\section{NP cytotoxicity}

After optimizing all the parameters, the cytotoxicity of the developed NPs was evaluated over human endothelial (Figure 4A) and breast cancer (Figure 4B) cells. Different concentrations of NPs were tested and, for concentrations 
(A)

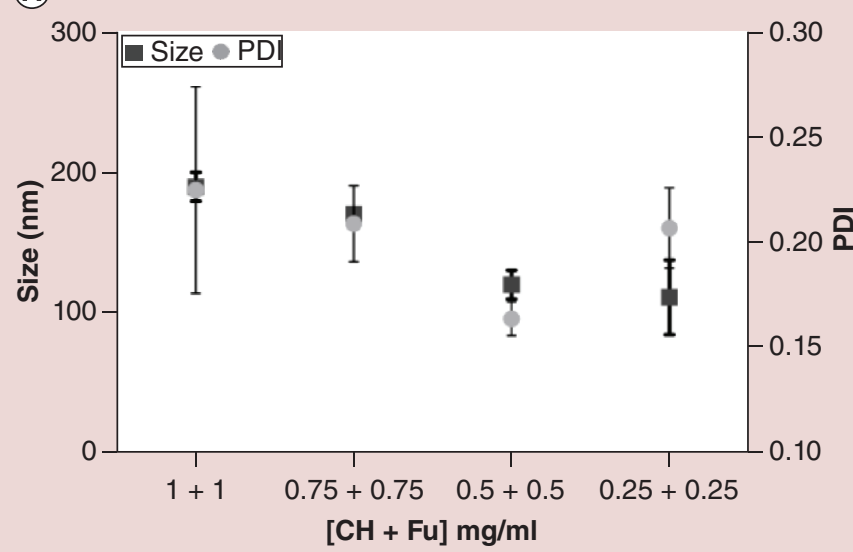

(C)

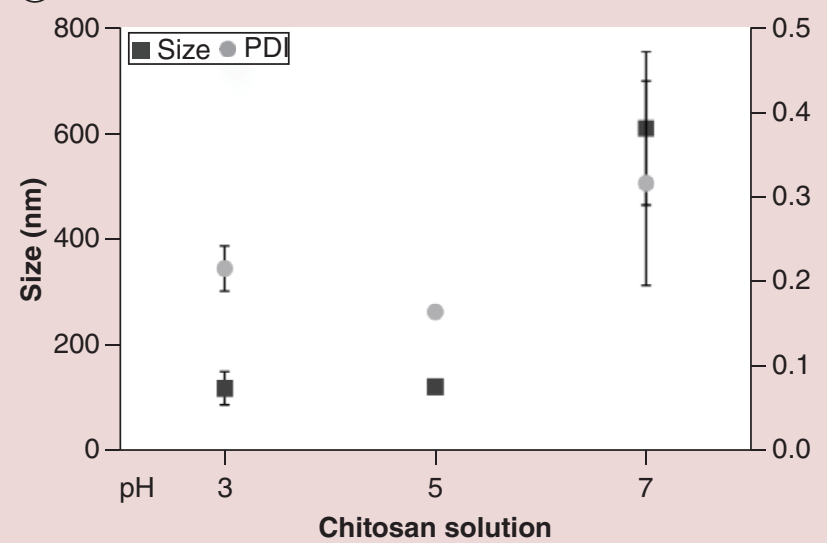

(E)

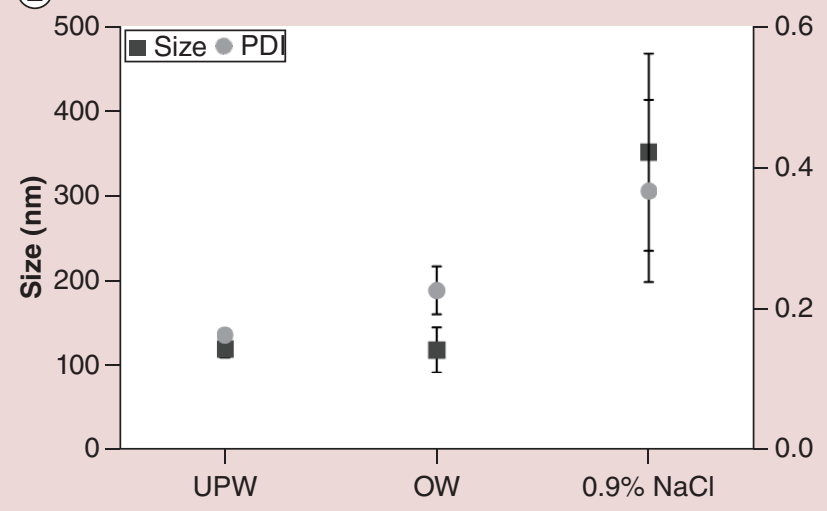

(B)

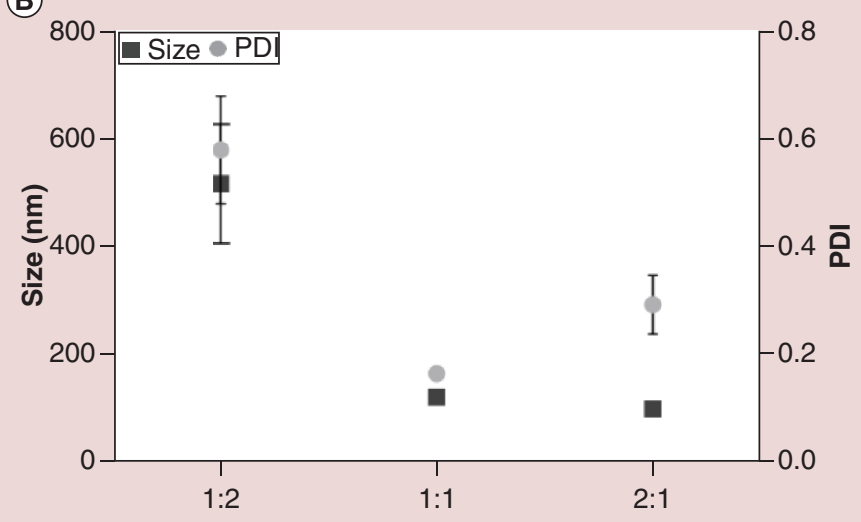

$[\mathrm{CH}+\mathrm{Fu}]$ ratio

(D)

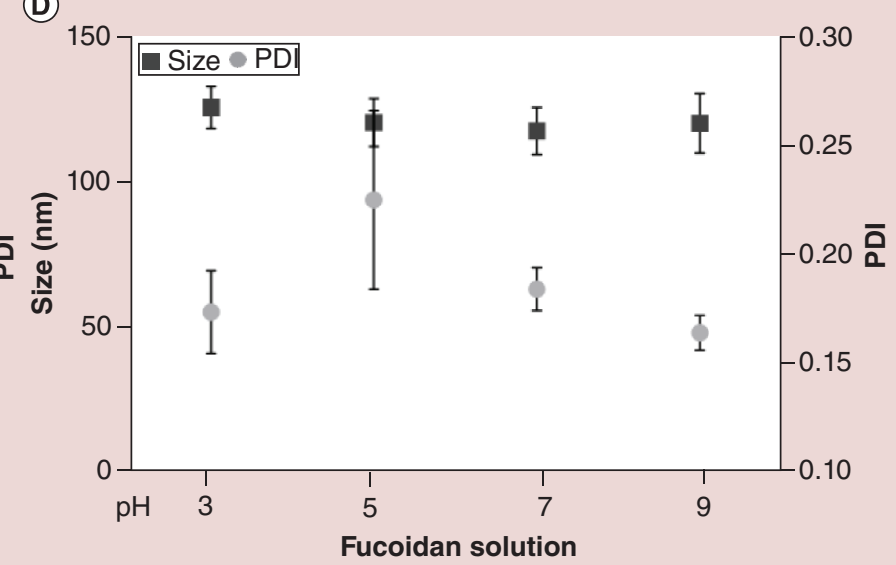

(F)

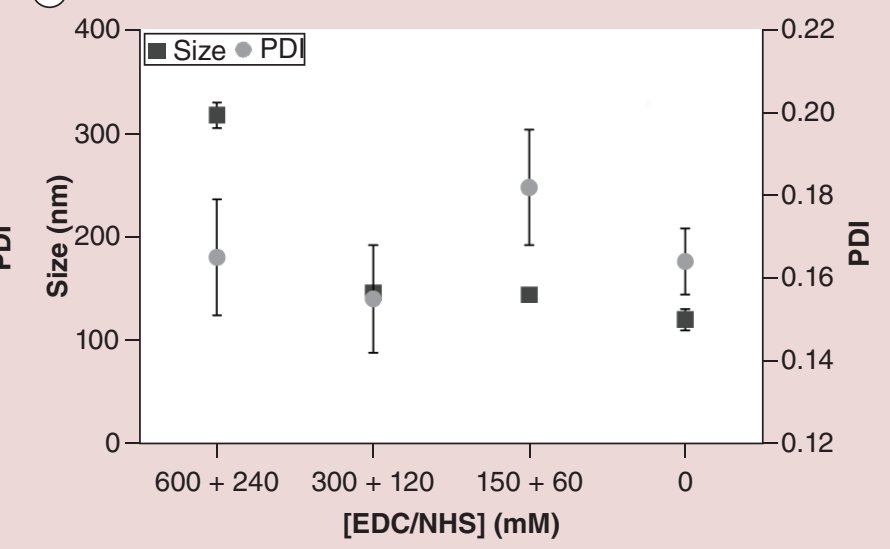

Figure 1. Nanoparticles' size and polydispersity index at different production parameters. (A \& B) Evaluation of $\mathrm{CH}$ and Fu concentration and ratio $(\mathrm{CH}+\mathrm{Fu})$, respectively. (C \& D) Evaluation of the influence of $\mathrm{CH}$ and Fu solutions' pH, respectively. (E) Influence of ionic strength and (F) Evaluation of different cross-linker (EDC/NHS) concentrations.

CH: Chitosan; EDC: 1-[3-(Dimethylamino)propyl]-3-ethylcarbodiimide hydrochloride; Fu: Fucoidan; PDI: Polydispersity index; NHS: $N$-hydroxysuccinimide.

above $8 \times 10^{9} \mathrm{NPs} / \mathrm{ml}$ they start to be toxic for both cell types, despite some significant differences in previous concentrations and time points. By SEM analysis, it was possible to observe the presence of the NPs at the surface of both cell types. 
(A)

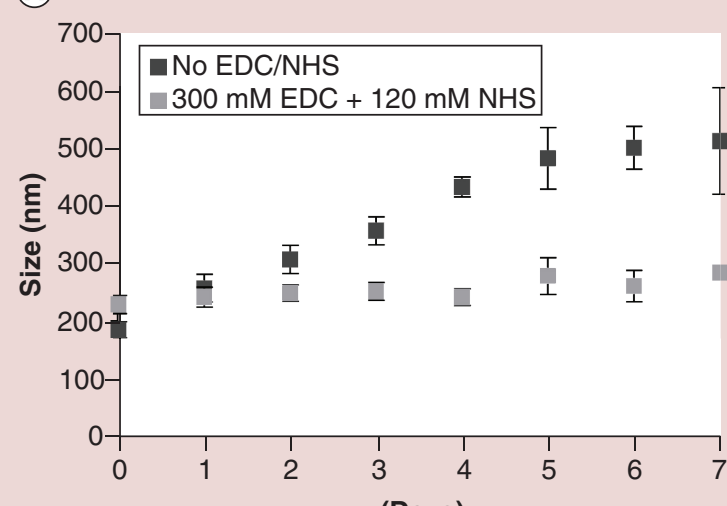

(C)

(Days)

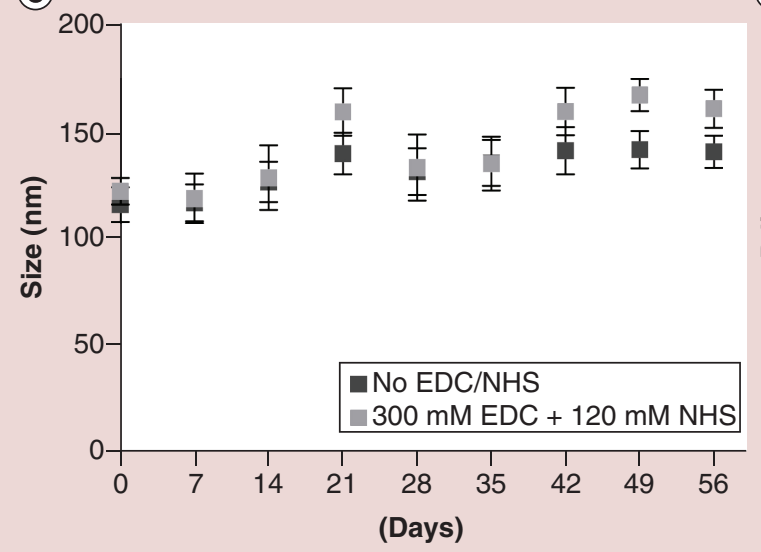

(B)

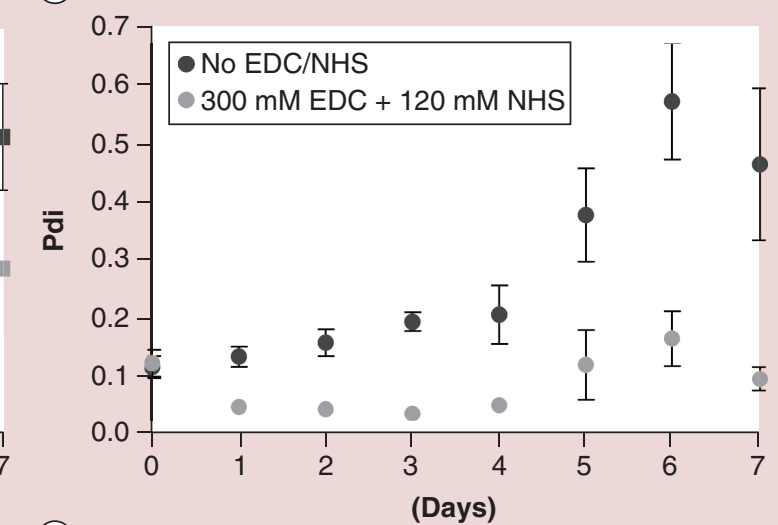

(D)

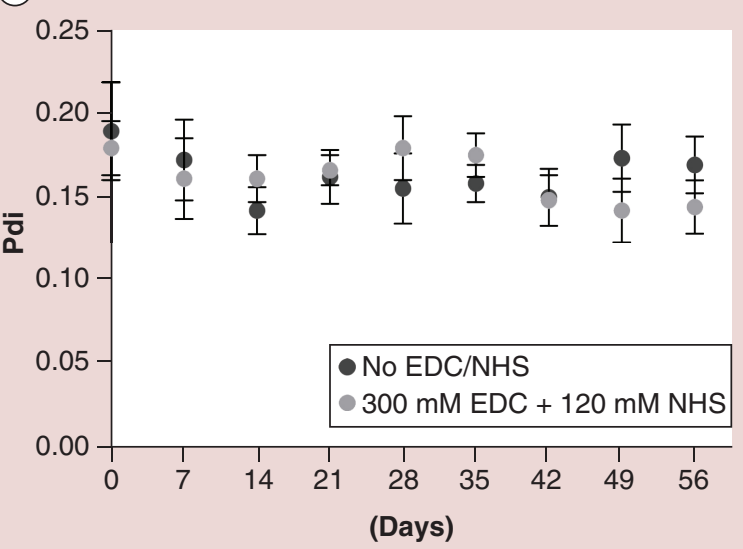

Figure 2. Nanoparticle stability. Size and polydispersity index of NPs at physiological $\left(37^{\circ} \mathrm{C}\right.$ and $\left.0.9 \% \mathrm{NaCl} ; \mathrm{A} \& \mathrm{~B}\right)$ and storage conditions ( $4^{\circ} \mathrm{C}$ and UPW; C \& D). (A \& C) Regard size and (B \& D) Regard PDI.

EDC: 1-[3-(Dimethylamino)propyl]-3-ethylcarbodiimide hydrochloride; NHS: N-hydroxysuccinimide; PDI: Polydispersity index; UPW: Ultrapure water.

\section{Table 1. Nanoparticles characterization by dynamic light scattering and nanoparticle tracking analysis.}

\begin{tabular}{|lll|}
\hline Technique & Property & Value \\
\hline DLS & Size $(\mathrm{nm})$ & $141 \pm 17$ \\
& PDI & $0.172 \pm 0.020$ \\
\hline NTA & Zeta potential $(\mathrm{mV})$ & $28.9 \pm 3.5$ \\
& Size $(\mathrm{nm})$ & $116 \pm 17$ \\
\hline & Concentration (particles/ml) & $7.8 \times 10^{10} \pm 1.2 \times 10^{10}$ \\
\hline
\end{tabular}

DLS: Dynamic light scattering; NTA: Nanoparticle tracking analysis; PDI: Polydispersity index.

Drug entrapment efficiency \& release studies

Gem entrapment efficiency

The drug entrapment efficiency was assessed by testing different Gem concentrations. Concentrations of 0.8 and $1.6 \mathrm{mg} / \mathrm{ml}$ present similar values and a maximum EE between 36 and 42\%, respectively. At higher concentrations, the system saturates and the $\mathrm{EE}$ decreases to values around $30 \%$.

In this sense, $0.8 \mathrm{mg} / \mathrm{ml}$ was the chosen concentration to evaluate the Gem-release profile, as well as the cytotoxic effects of NPs_Gem over human endothelial and breast cancer cells. NPs_Gem presented a size around $134 \mathrm{~nm}$ and a PDI of 0.186 (Figure 5B), similar to the values observed for the nonloaded NPs (Table 1). 


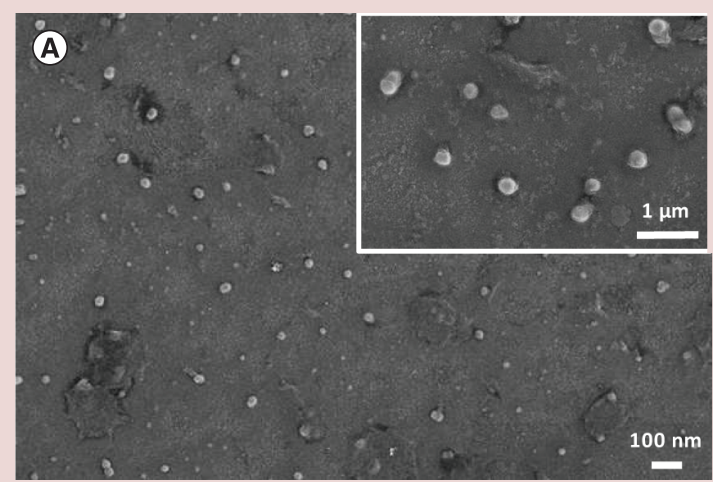

(B)

Figure 3. Nanoparticles morphology. Observation by scanning electron microscopy (SEM; A) and scanning transmission electron microscopy (STEM; B).

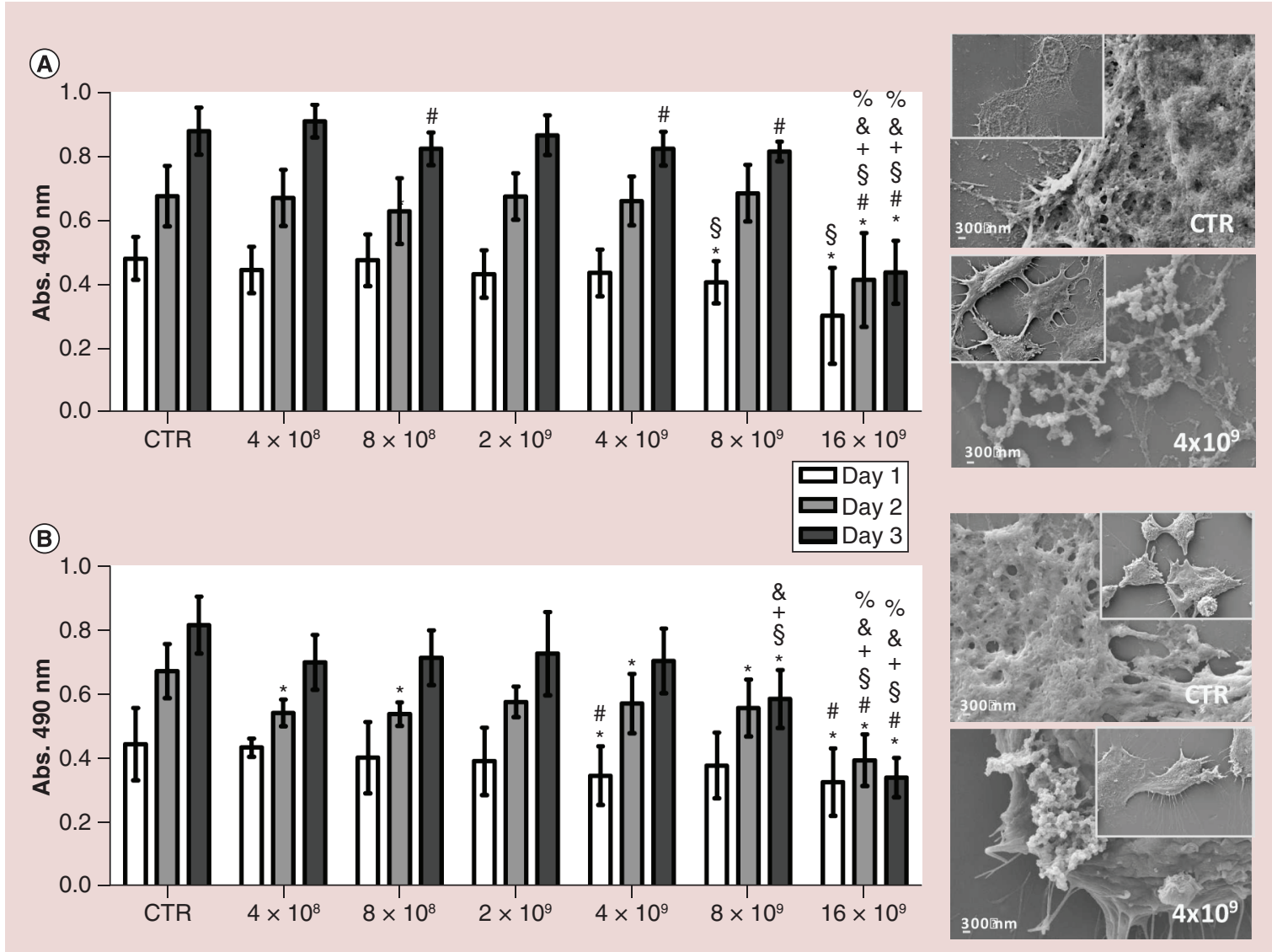

Figure 4. Cytotoxicity of developed NPs. Influence of NPs concentrations over human endothelial (A) and breast cancer (B) cells at different time points (1, 2 and 3 days). Data were considered statistically significant at $p<0.05$. ${ }^{*}$ Indicates significant differences compared with CTR; Compared with $4 \times 10^{8} ;$ \& Compared with $8 \times 10^{8} ;{ }^{+}$Compared with $2 \times 10^{9} ;$ \& Compared with $4 \times 10^{9}$ and ${ }^{\%}$ Compared with $8 \times 10^{9}$. SEM observation of control and $4 \times 10^{9} \mathrm{NPs} / \mathrm{ml}$ culture conditions at day 3.

CTR: Control; NP: Nanoparticle; SEM: Scanning electron microscopy. 


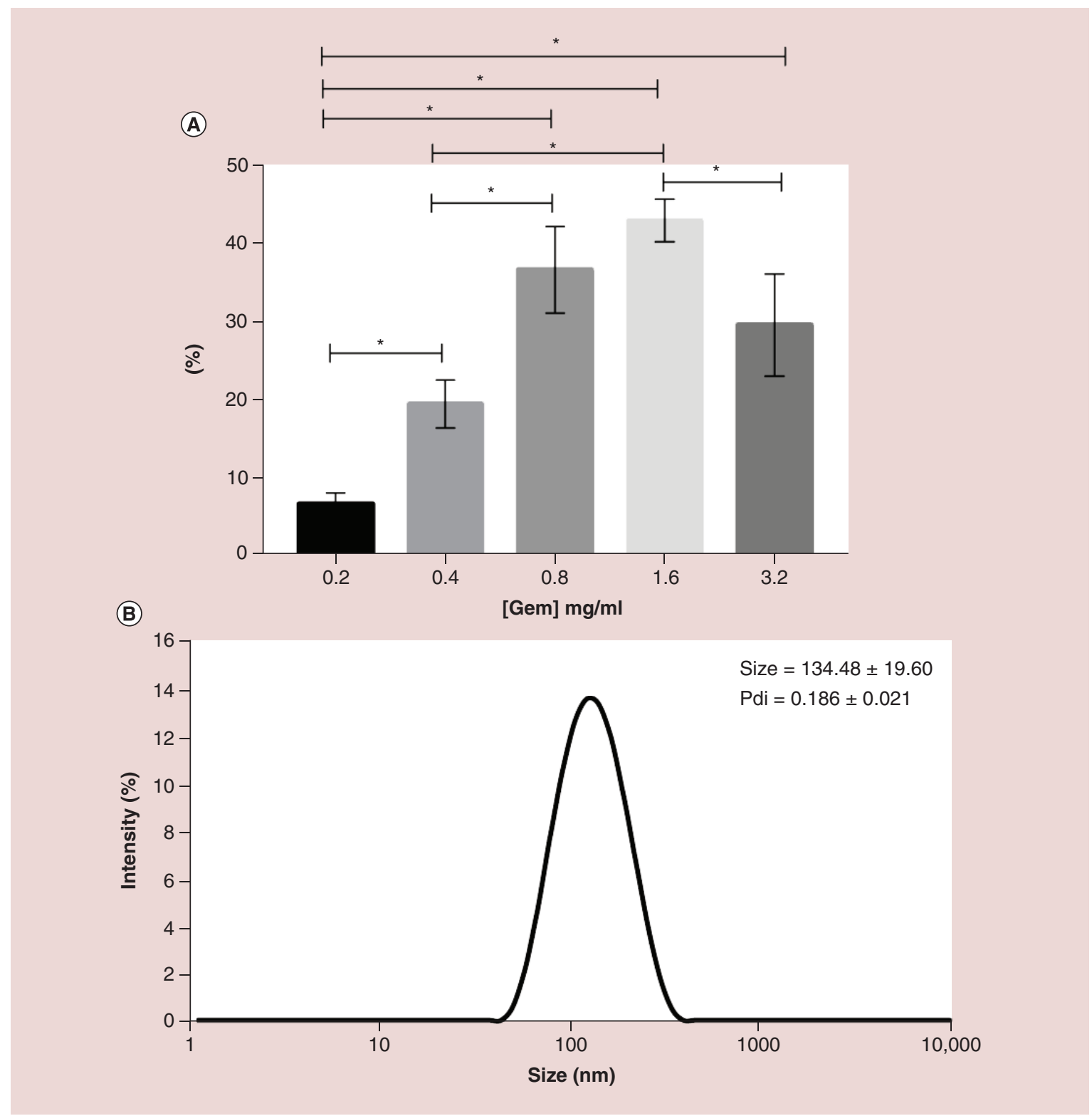

Figure 5. Gem-loaded NPs. Entrapment efficiency (A) and size distribution representation (B). Gem: Gemcitabine; NP: Nanoparticle.

\section{Gem-release profile}

The release of Gem from marine-origin polymeric NPs was evaluated at $37^{\circ} \mathrm{C}$ and $0.9 \% \mathrm{NaCl}$ to mimic physiological conditions. Figure 6 shows that the developed system releases around $84 \%$ of the drug in a sustained way during $4 \mathrm{~h}$, then maintaining a steady-state profile for up to $72 \mathrm{~h}$.

\section{Cytotoxic effects of drug-loaded NPs over cancer \& noncancer cells}

NPs at $4 \times 10^{9} \mathrm{NPs} / \mathrm{ml}$ do not present statistical significant differences when compared with the control condition for both endothelial and breast cancer cells, in all time points evaluated (Figure 7), confirming the results already reported in Figure 4. However, NPs_Gem are cytotoxic for breast cancer cells since the first day, but being also cytotoxic at days 2 and 3 for endothelial cells. Free Gem also presents cytotoxicity to endothelial cells with similar values to the ones observed with NPs_Gem. Concerning breast cancer cells, NPs_Gem present increased toxicity at 


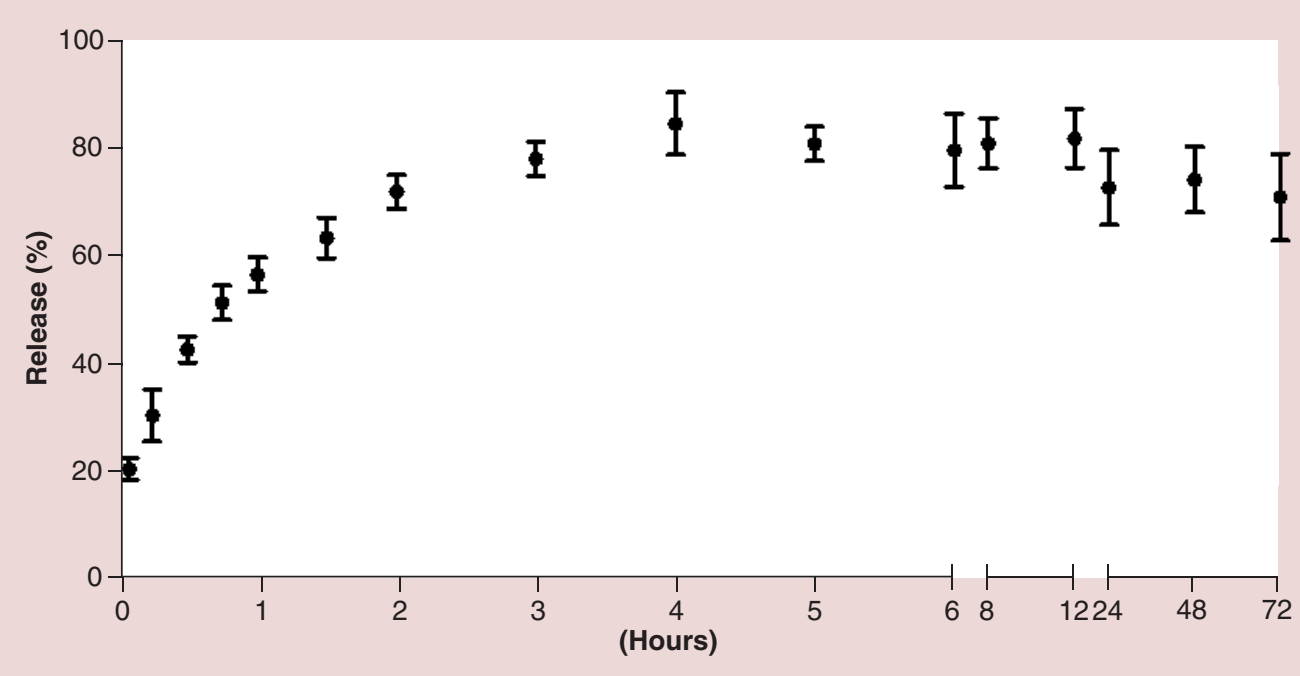

Figure 6. Gemcitabine-release profile from marine-origin polymeric nanoparticles $\left(0.9 \% \mathrm{NaCl}, 37^{\circ} \mathrm{C}, 60 \mathrm{rpm}\right)$.
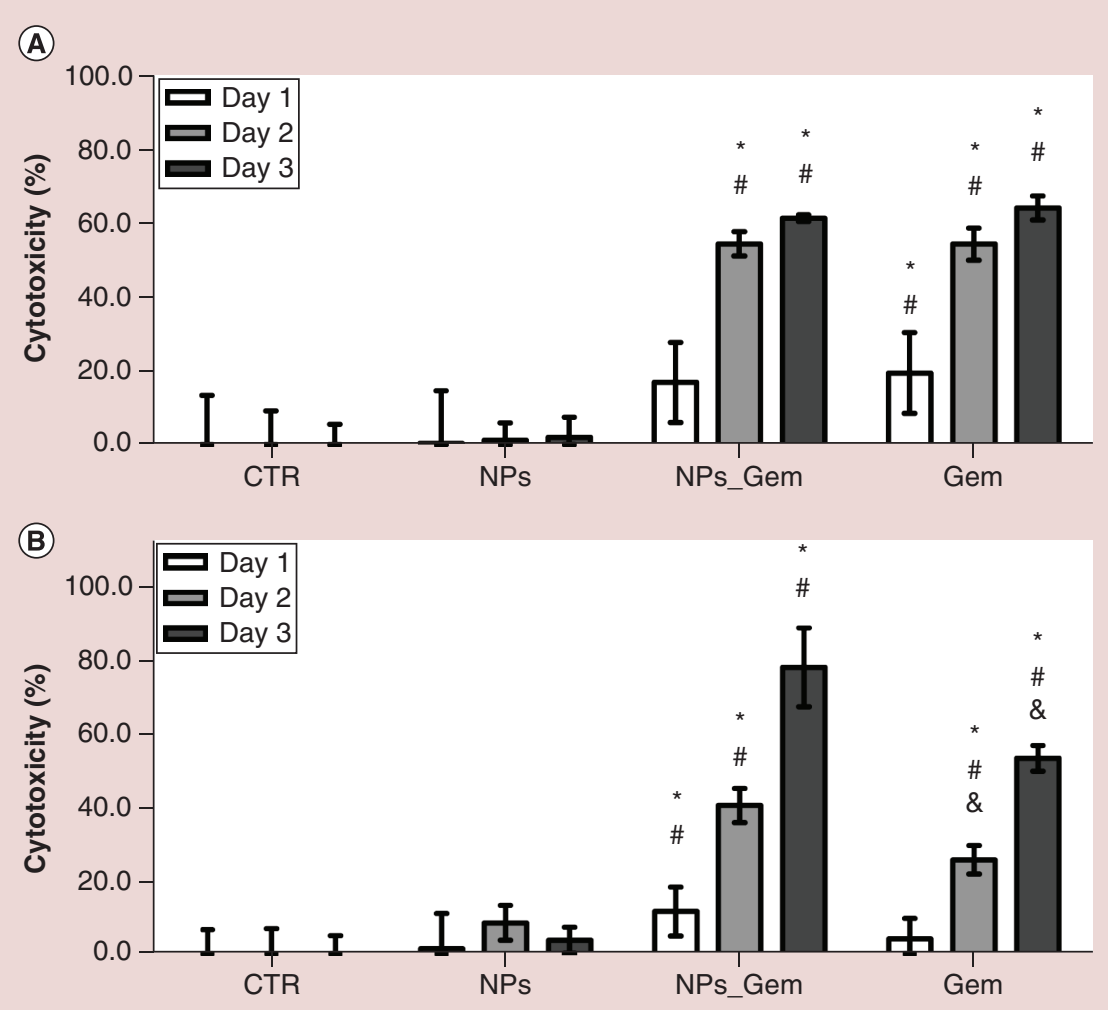

Figure 7. Cytotoxicity of developed Gem-loaded NPs. Influence over human endothelial (A) and breast cancer (B) cells at different time points (1, 2 and 3 days). Data were considered statistically significant at $p<0.05$. *Indicates significant differences compared with CTR; \# Compared with NPs; ${ }^{\text {}}$ Compared with NPs_Gem.

CTR: Control; NP: Nanoparticle; NP_Gem: Gemcitabine-loaded NP. 
day $2(15 \%)$ and at day 3 (25\%) when comparing with free Gem. Furthermore, NP_Gem presents higher toxicity over breast cancer than endothelial cells at day $3(17 \%)$.

\section{Discussion}

Polyelectrolyte complexation has been widely reported due to its cost-effectiveness and easy production of NPs. It takes advantage of the interactions between two oppositely charged polymers and a spontaneous formation of the NPs occurs [25]. There are different parameters described to affect NPs size and PDI, namely the polymer concentration and ratio, polymer solution $\mathrm{pH}$ and ionic strength [26,27]. Our results showed that by decreasing polymers concentration, it was possible to obtain similar NPs.

However, higher PDI values are observed for both the high and low concentrations, showing obtainance of NPs with a nonhomogenous size distribution. Thus, it seems that at higher concentrations there would be high entropy and at lower concentrations reduced interactions between oppositely charged polymers. The influence of polymers ratio was also evaluated over NPs' size and PDI. When higher amounts of Fu are present (CH:Fu ratio 1:2), the NPs present an increased size and PDI, showing that probably there is the formation of polymeric aggregates instead of NPs. Indeed, the amino groups of $\mathrm{CH}$ have a pKa of approximately 6.5, which leads to a protonation in acidic conditions. In solutions with $\mathrm{pH}>6.5$, most amino groups would be deprotonated and $\mathrm{CH}$ tends to precipitate [28]. Being deprotonated, the amine groups will be less available to form a complex with $\mathrm{Fu}$, resulting in a less controlled process, with the participation of other types of interactions gaining relevance, which will result in higher variability and consecutively high values of size and PDI, outside the range to be considered as NPs. CH at acidic pHs present protonated amines being as these chemical groups are more prone to form polyelectrolyte complexes with a negatively charged polymer $(\mathrm{Fu})$, with ionic attractive interactions ruling the process, leading to the formation of the desired NPs. Despite similar size values for the three other $\mathrm{pHs}$, $\mathrm{pH} 5$ was the one that presented more homogeneous NPs' size. Fu solution $\mathrm{pH}$ seems not to influence the development of NPs size, although a low PDI value is observed when the $\mathrm{pH}$ is not adjusted ( $\mathrm{pH}$ 9; Figure 1C \& D). Ionic strength was analyzed by dissolving the polymers in different solvents, UPW being the one that presents the better outcomes in terms of size and PDI. As expected, $0.9 \% \mathrm{NaCl}$ tends to form bigger and nonhomogenous NPs due to the presence of higher amounts of ions, which would have a charge shielding effect over the protonated groups in polyelectrolytes and thus decreasing the relevance of ionic interactions in the assembly process. Fu/CH NPs may become unstable at physiological conditions due to the deprotonation of $\mathrm{CH}$ amine groups. Trying to enhance NPs stability, a cross-linking agent was tested: EDC/NHS. EDC is often used to cross-link amines $\left(\mathrm{NH}_{2}\right)$ to carboxylate groups, whose activity is enhanced by NHS, through activation of the carboxylate group [29]. In the present case, the rationale is to cross-link the amine groups of $\mathrm{CH}$ with the carboxylic group of the uronic acids that are present in Fu structure [8]. Different concentrations of EDC/NHS were evaluated and the $300 \mathrm{mM} \mathrm{EDC}+120 \mathrm{mM}$ NHS presented the smaller PDI. Despite $150 \mathrm{mM} \mathrm{EDC}+60 \mathrm{mM}$ NHS present similar results, these concentrations presented higher PDI, reason why a higher concentration was chosen to continue the study. Higher concentrations $(600 \mathrm{mM}$ EDC $+240 \mathrm{mM}$ NHS) seem to start precipitating and affecting NPs' size, increasing it to around $300 \mathrm{~nm}$. NPs final formulation was set as: $0.5 \mathrm{mg} / \mathrm{ml}$ polymers concentration, $1: 1$ ratio, $\mathrm{pH} 5(\mathrm{CH})$ and $\mathrm{pH} 9(\mathrm{Fu})$ and UPW as the polymers solvent.

After this optimization process and setting all the parameters, two conditions were chosen to conduct the stability assays: one without cross-linking and the other cross-linked. NPs stability at physiological and storage conditions was assessed. Physiological conditions were mimetized by diluting the produced NPs in $0.9 \% \mathrm{NaCl}$, which have an osmolarity similar to circulating plasma 285-295 mOsm/1 [30]. Both NPs increase their size to around $200 \mathrm{~nm}$, when in contact with this isotonic solution, due to an increase in the ionic strength with associated charge shielding but also higher water uptake (Donnan effect) [31]. Cross-linked NPs present a quite stable profile along the 7 days, whereas from day 3 on, the noncross-linked NPs start to increase their size, indicating that they probably start to disintegrate at physiological osmolarity. We proceed the characterization of cross-linked NPs that present a size around $140 / 115 \mathrm{~nm}$ (DLS/NTA), a PDI of 0.172, which indicates that these NPs are quite homogeneous and a zeta potential of $+29 \mathrm{mV}$ indicating that $\mathrm{CH}$ is at the surface of NPs. By NTA, the NPs presented a smaller size due to the fact that NTA is a more precise technique than DLS [32]. By SEM and STEM observation, we were able to confirm the round morphology of the NPs, with a size smaller than $100 \mathrm{~nm}$. The difference between DLS/NTA measurements and SEM/STEM is due to the fact that in the first characterization, NPs are measured in wet state, whereas for SEM/STEM, NPs are analyzed in a dry state. 
After all the optimization steps, the cytotoxicity of the developed marine-origin polymeric NPs was evaluated over human endothelial and breast cancer cells at different concentrations. NPs start to become more toxic to both cell types at the highest concentrations $\left(8 \times 10^{9}\right.$ and $\left.16 \times 10^{9} \mathrm{NPs} / \mathrm{ml}\right)$ tested. From SEM observation, we were able to observe NPs at the surface of both cell types. From these results, $4 \times 10^{9} \mathrm{NPs} / \mathrm{ml}$ concentration (nontoxic concentration) was selected to conduct the further experiments.

Breast cancer is sensitive to several cytotoxic drugs that may be used as single or combined therapies. In the past years, new cytotoxic agents have been developed and approved to be used in cancer treatments. Gem has shown antitumoral activity and good toxicity profiles to be used as single-agent therapy for advanced breast cancer. One of the drawbacks of using Gem by intravenous or oral administration is related with its rapid inactivation by enzymatic delamination, low bioavailability and short half-life. These problems require an increase in dosage that may lead to unwanted side effects [19-22]. The use of NPs may overcome some of these limitations since they will be able to protect the drug from enzymatic degradation, diminishing the above-mentioned efficiency problems [33].

The loading of Gem on the marine-origin polymeric NPs was evaluated by testing different Gem concentrations. In Figure 5A, it is possible to observe that, by increasing the concentration of the drug, there is an increase in the $\mathrm{EE}$ until it reaches a maximum concentration of $1.6 \mathrm{mg} / \mathrm{ml}(\mathrm{EE}=42 \%)$. At $3.2 \mathrm{mg} / \mathrm{ml}$, the system saturates and the EE decreases to around 30\%. NPs_Gem were characterized in terms of size and PDI, showing no significant differences. The release profile was assessed at $37^{\circ} \mathrm{C}$ and $0.9 \% \mathrm{NaCl}$ to mimic physiological conditions. From the stability studies, it was observed that the size of the NPs increased, due to the ionic strength of the solution that weakens the electrostatic interactions, leading to the release of Gem from the NPs [10]. Around 84\% of Gem was released in the first $4 \mathrm{~h}$ in a sustained way, until reaching a steady state.

To assess the antitumoral activity of the developed DDS, it is important to have in mind the tumor microenvironment that comprises not only cancer cells but also noncancer cells (e.g., endothelial, fibroblasts). Indeed, as NPs_Gem are intended to be injected intravenously, the first barrier they will face is the blood vessels wall and thus endothelial cells. Different Fu-based NPs have been developed as anticancer strategies. As example, Fu-cistaplin NPs have been developed and evaluated for colon cancer [34], whereas Fu encapsulated into liposomes showed anticancer activity against osteosarcoma [35]. In another attempt, doxorubicin was incorporated into a protamine $/ \mathrm{Fu}$ system and these loaded NPs improved inhibitory effects against a breast cancer cell line [36]. However, as far as we know there are no studies in the literature that report the use of Fu to develop NPs for the delivery of Gem. On the other hand, CH-based NPs have been studied for the delivery of antitumoral agents, namely for the delivery of Gem. For example, the loading of Gem into $\mathrm{CH}$ magnetic NPs increased Gem efficacy when compared with free Gem [37]. In other study, CH-based NPs were prepared by layer-by-layer technique for the incorporation of Gem and platinum aiming at lung cancer treatments [38]. Other studies reported the use of CH-based NPs for the delivery of other antitumor drugs: PEG-layered CH NPs enhanced the efficacy of ormeloxifene in breast cancer [39] and platinum-gold NPs with $\mathrm{CH}$ and doxorubicin presented cell-specific cytotoxicity [40].

In this sense, the combination of these two marine-origin polymers to develop NPs for the delivery of Gem is a novel process with potential positive outcome. We evaluated the Gem loaded-NPs toxicity over human endothelial and breast cancer cell lines. Regarding endothelial cells, both NPs_Gem and free Gem present similar cytotoxicity values. However, concerning breast cancer cells, NPs_Gem increased toxic effects of around 15 and $25 \%$ at days 2 and 3, respectively, when compared with free Gem. At day 3, when comparing breast cancer and endothelial cells, it was observed an increased cytotoxicity of around $17 \%$ over breast cancer cells. The increased cytotoxicity of the NPs_Gem when compared with free Gem may be attributed to the cellular trafficking pathway, suggesting a more effective uptake of the drug by breast cancer cells. The encapsulation of cisplatin into CH lipid NPs was reported, demonstrating increased apoptosis in cancer cells when compared with free cisplatin [41]. A recent paper reported that $\mathrm{CH}$ NPs loaded with curcumin reduced the drug uptake in normal cells, while the same was internalized into cancer cells [42]. Another study reported the internalization of $\mathrm{CH}$-coated NPs loaded with doxorubicin into breast cancer cells cytoplasm and nucleus. The authors stated that the uptake of these NPs may be attributed to the positive charge of their surface. That positively charged CH NPs can have charge-based interaction with the cell membrane and may enter cells either by direct penetration or by endocytosis [43]. Thus, although not presenting a protective effect against the toxicity over endothelial cells, the Fu/CH NPs exhibit a synergistic effect with Gem resulting in a higher toxicity over breast cancer cells. Moreover, these results confirm that, by encapsulating Gem into an NP, the drug is protected from enzymatic degradation, increasing its half-life and biological response. 


\section{Conclusion}

Marine-origin polymeric NPs were successfully produced by polyelectrolyte complexation, presenting increased stability at physiological conditions when cross-linked with EDC/NHS. Gem was encapsulated in the developed NP system. This DDS increased toxicity over human breast cancer cells, when compared with free Gem, without increasing their toxic effect over endothelial cells. Taken together, the herein-developed DDS is an interesting strategy to be further explored on breast cancer therapies.

\section{Summary points}

Nanoparticle production \& characterization

- Nanoparticles (NPs) were successfully produced by polyelectrolyte complexation, presenting a size around $115-140 \mathrm{~nm}$.

- NPs present a polydispersity index less than 0.2 , demonstrating NPs' size homogeneity.

- Cross-linked NPs presented increased stability at physiological conditions than noncross-linked NPs.

Gemcitabine entrapment efficiency \& release profile

- The maximum entrapment efficiency of the system is around $42 \%$ for $1.6 \mathrm{mg} / \mathrm{ml}$ (above this concentration, the system saturates).

- The system releases around $84 \%$ of the drug in the first $4 \mathrm{~h}$.

Drug-loaded NPs cytotoxicity

- Drug-loaded NPs presented increased toxicity (around $25 \%$ at day 3 ) over human breast cancer cells when compared with free gemcitabine.

- Drug-loaded NPs did not present increased toxicity over endothelial cells when compared with free gemcitabine.

- Drug-loaded NPs present higher toxicity over breast cancer cells (around 17\%) when compared with endothelial cells.

\section{Acknowledgements}

The authors acknowledge Carla Silva and Artur Cavaco-Paulo for the help using NanoSight NS500 instrument.

\section{Financial \& competing interests disclosure}

The authors thank the PhD scholarship of C Oliveira for 'NORTE-08-5369-000037' financed by NORTE 2020, Portuguese Foundation for Science and Tecnology (FCT) for the investigator grant of A Martins for (IF/00376/2014) and the support from European Research Council under the Advanced Grant ComplexiTE. The work here reported also received financial support from the European Regional Development Fund (ERDF) under the Structured Project 'Accelerating tissue engineering and personalized medicine discoveries by the integration of key enabling nanotechnologies, marine-derived biomaterials and stem cells' (NORTE-01-0145FEDER-000021), supported by Norte Portugal Regional Operational Program (NORTE 2020), under the PORTUGAL 2020 Partnership Agreement. The authors have no other relevant affiliations or financial involvement with any organization or entity with a financial interest in or financial conflict with the subject matter or materials discussed in the manuscript apart from those disclosed.

No writing assistance was utilized in the production of this manuscript.

\section{References}

Papers of special note have been highlighted as: $\bullet$ of interest

1. Siegel R, Ma J, Zou Z, Jemal A. Cancer statistics, 2014. CA Cancer J. Clin. 64(1), 9-29 (2014).

2. Desantis C, Ma J, Bryan L, Jemal A. Breast cancer statistics, 2013. CA Cancer J. Clin. 64(1), 52-62 (2014).

3. Aftimos PG, Gombos A, Pugliano L, Awada A, Piccart MJ. Breast cancer. In: Side Effects of Medical Cancer Therapy: Prevention and Treatment. Dicato MA (Ed.). Springer, London, UK 29-117 (2013).

4. Moyad MA. Rapid review of breast cancer treatment side effects and dietary supplement/integrative options from A to Z: what helps, harms, or does nothing? In: Integrative Medicine for Breast Cancer: An Evidence-Based Assessment. Moyad MA (Ed.) Springer International Publishing, Basel, Switzerland 225-342 (2016).

5. Silva TH, Alves A, Ferreira BM et al. Materials of marine origin: a review on polymers and ceramics of biomedical interest. Int. Mater. Rev. 57(5), 276-306 (2012).

6. Atashrazm F, Lowenthal RM, Woods GM, Holloway AF, Dickinson JL. Fucoidan and cancer: a multifunctional molecule with anti-tumor potential. Mar. Drugs 13(4), 2327-2346 (2015).

- Relevance of mechanisms of action of fucoidan (Fu) and its antitumoral activity. 
7. Fitton JH, Stringer DN, Karpiniec SS. Therapies from fucoidan: an update. Mar. Drugs 13(9), 5920-5946 (2015).

8. Oliveira C, Ferreira AS, Novoa-Carballal R et al. The key role of sulfation and branching on fucoidan antitumor activity. Macromol. Biosci. 17(5), doi:10.1002/mabi.201600340 (2017) (Epub ahead of print).

- Chemical structure and its influence in Fu antitumoral activity.

9. Chollet L, Saboural P, Chauvierre C, Villemin J-N, Letourneur D, Chaubet F. Fucoidans in nanomedicine. Mar. Drugs 14(8), 145 (2016).

10. Lu K-Y, Li R, Hsu C-H et al. Development of a new type of multifunctional fucoidan-based nanoparticles for anticancer drug delivery. Carbohydr. Polym. 165, 410-420 (2017).

- Development and characterization of Fu-based nanoparticles (NPs) tested with MDA-MB-231 breast cancer cells.

11. Huang YC, Yang YT. Effect of basic fibroblast growth factor released from chitosan-fucoidan nanoparticles on neurite extension. J. Tissue Eng. Regen. Med. 10(5), 418-427 (2016).

12. Huang Y-C, Chen J-K, Lam UI, Chen S-Y. Preparing, characterizing, and evaluating chitosan/fucoidan nanoparticles as oral delivery carriers. J. Polym. Res. 21(5), 415 (2014).

13. Huang Y-C, Li R-Y. Preparation and characterization of antioxidant nanoparticles composed of chitosan and fucoidan for antibiotics delivery. Mar. Drugs 12(8), 4379-4398 (2014).

14. Liu Z, Jiao Y, Wang Y, Zhou C, Zhang Z. Polysaccharides-based nanoparticles as drug delivery systems. Adv. Drug Deliv. Rev. 60(15), 1650-1662 (2008).

15. Masood F. Polymeric nanoparticles for targeted drug delivery system for cancer therapy. Mater. Sci. Eng. C. Mater. Biol. Appl. 60, 569-578 (2016).

16. Hayashi H, Kurata T, Nakagawa K. Gemcitabine: efficacy in the treatment of advanced stage nonsquamous non-small cell lung cancer. Clin. Med. Insights Oncol. 5, 177-184 (2011).

17. Kawaguchi $\mathrm{H}$, Terai $\mathrm{Y}$, Tanabe A et al. Gemcitabine as a molecular targeting agent that blocks the Akt cascade in platinum-resistant ovarian cancer. J. Ovarian Res. 7, 38-38 (2014).

18. O'reilly EM, Roach J, Miller P et al. Safety, pharmacokinetics, pharmacodynamics, and antitumor activity of necuparanib combined with nab-paclitaxel and gemcitabine in patients with metastatic pancreatic cancer: Phase I results. Oncologist doi:10.1634/theoncologist.2017-0472 (2017) (Epub ahead of print).

19. Hertel LW, Boder GB, Kroin JS et al. Evaluation of the antitumor activity of gemcitabine (2',2'-difluoro-2'-deoxycytidine). Cancer Res. 50(14), 4417-4422 (1990).

20. Modi S, Seidman AD. Single-agent gemcitabine in the treatment of advanced breast cancer. Clin. Breast Cancer 4(Suppl. 3) S101-S106 (2004).

21. Heinemann V. Role of gemcitabine in the treatment of advanced and metastatic breast cancer. Oncology 64(3), 191-206 (2003).

- Gemcitabine and its role in breast cancer, to better understand the mechanism behind its biological activity.

22. Hortobagyi GN. Treatment of advanced breast cancer with gemcitabine and vinorelbine. Oncology (Williston Park) 15(2 Suppl. 3), 15-17 (2001).

23. Santiago T, Devaux RS, Kurzatkowska K, Espinal R, Herschkowitz JI, Hepel M. Surface-enhanced Raman scattering investigation of targeted delivery and controlled release of gemcitabine. Int. J. Nanomedicine 12, 7763-7776 (2017).

24. Smith M, Hepel M. Controlled release of targeted anti-leukemia drugs azacitidine and decitabine monitored using surface-enhanced Raman scattering (SERS) spectroscopy. Med. J. Chem. 6(4), 125 (2017).

25. Lima AC, Sher P, Mano JF. Production methodologies of polymeric and hydrogel particles for drug delivery applications. Expert Opin. Drug Deliv. 9(2), 231-248 (2012).

26. Venkatesan J, Anil S, Kim S-K, Shim M. Seaweed polysaccharide-based nanoparticles: preparation and applications for drug delivery. Polymers 8(2), 30 (2016).

27. Lee EJ, Lim K-H. Formation of chitosan-fucoidan nanoparticles and their electrostatic interactions: quantitative analysis. J. Biosci. Bioeng. 121(1), 73-83 (2016).

- Analysis of the mechanism involved in the production of polymeric NPs by polyelectrolyte complexation.

28. Kim S-K. Chitin and Derivatives: Advances in Drug Discovery and Developments. CRC Press, Florida, USA 527, (2013).

29. Staros JV, Wright RW, Swingle DM. Enhancement by N-hydroxysulfosuccinimide of water-soluble carbodiimide-mediated coupling reactions. Anal. Biochem. 156(1), 220-222 (1986).

30. Wahed ADA. Water, homeostasis, electrolytes, and acid-base balance. In: Clinical Chemistry, Immunology and Laboratory Quality Control. Elsevier, New York, USA, 67-84 (2014).

31. Jiang J, Oberdörster G, Biswas P. Characterization of size, surface charge, and agglomeration state of nanoparticle dispersions for toxicological studies. J. Nanoparticle Res. 11(1), 77-89 (2009). 
32. Filipe V, Hawe A, Jiskoot W. Critical evaluation of nanoparticle tracking analysis (NTA) by NanoSight for the measurement of nanoparticles and protein aggregates. Pharm. Res. 27(5), 796-810 (2010).

33. Derakhshandeh K, Fathi S. Role of chitosan nanoparticles in the oral absorption of gemcitabine. Int. J. Pharmaceut. 437(1), 172-177 (2012).

34. Hwang P-A, Lin X-Z, Kuo K-L, Hsu F-Y. Fabrication and cytotoxicity of fucoidan-cisplatin nanoparticles for macrophage and tumor cells. Materials 10(3), 291 (2017).

- Synthesis and characterization of Fu/cistaplin NPs as a drug-delivery system for anticancer therapies.

35. Kimura R, Rokkaku T, Takeda S, Senba M, Mori N. Cytotoxic effects of fucoidan nanoparticles against osteosarcoma. Mar. Drugs 11(11), 4267-4278 (2013).

36. Lin YH, Lu KY, Tseng CL, Wu JY, Chen CH, Mi FL. Development of genipin-crosslinked fucoidan/chitosan-N-arginine nanogels for preventing Helicobacter infection. Nanomedicine (Lond.) 12(12), 1491-1510 (2017).

37. Parsian M, Unsoy G, Mutlu P, Yalcin S, Tezcaner A, Gunduz U. Loading of Gemcitabine on chitosan magnetic nanoparticles increases the anti-cancer efficacy of the drug. Eur. J. Pharmacol. 784(Supplement C), 121-128 (2016).

38. Zhang R, Ru Y, Gao Y, Li J, Mao S. Layer-by-layer nanoparticles co-loading gemcitabine and platinum (IV) prodrugs for synergistic combination therapy of lung cancer. Drug Des. Devel. Ther. 11, 2631-2642 (2017).

39. Agrawal S, Ahmad H, Dwivedi M et al. PEGylated chitosan nanoparticles potentiate repurposing of ormeloxifene in breast cancer therapy. Nanomedicine (Lond.) 11(16), 2147-2169 (2016).

- Development of chitosan-based NPs for the delivery of anticancer agents increased toxicity over breast cancer cell lines.

40. Maney V, Singh M. An in vitro assessment of novel chitosan/bimetallic PtAu nanocomposites as delivery vehicles for doxorubicin. Nanomedicine (Lond.) 12(21), 2625-2640 (2017).

41. Wang JY, Wang Y, Meng X. Chitosan nanolayered cisplatin-loaded lipid nanoparticles for enhanced anticancer efficacy in cervical cancer. Nanoscale Res. Lett. 11(1), 524 (2016).

42. Abruzzo A, Zuccheri G, Belluti F et al. Chitosan nanoparticles for lipophilic anticancer drug delivery: development, characterization and in vitro studies on HT29 cancer cells. Colloids Surf. B Biointerfaces 145, 362-372 (2016).

43. Kulkarni VM, Bodas D, Dhoble D, Ghormade V, Paknikar K. Radio-frequency triggered heating and drug release using doxorubicin-loaded LSMO nanoparticles for bimodal treatment of breast cancer. Colloids Surf. B Biointerfaces 145, 878-890 (2016). 